\title{
The immediate effects of whole body vibration on cervical joint position sense in subjects with forward head posture
}

\author{
Arepheh Salami ${ }^{1}$ \\ Mohammad Mohsen Roostayi ${ }^{1}$ \\ Sedigheh Sadat Naimi ${ }^{1}$ \\ Azadeh Shadmehr ${ }^{2}$ \\ Alireza Akbarzadeh Baghban ${ }^{3}$ \\ 1 Physiotherapy Research Center, School of \\ Rehabilitation, Shahid Beheshti University \\ of Medical Sciences, \\ Tehran, Iran \\ 2 Research \& International affairs of School of Reha- \\ bilitation, Teheran University of Medical Sciences, \\ Teheran, Iran \\ 3 Proteomics Research Centre, Department of Basic \\ Sciences, School of Rehabilitation Sciences, \\ Shahid Beheshti University of Medical Sciences, \\ Tehran, Iran
}

Corresponding author:

Mohammad Mohsen Roostayi

Physiotherapy Research Center, School

of Rehabilitation

Shahid Beheshti University of Medical Sciences,

Tehran, Iran

E-mail: roosta@sbmu.ac.ir

\section{Summary}

Forward head posture (FHP) is considered to be one of the most common postural disorders in the spinal column. Recently studies have reported impaired proprioception to be one of the results of prolonged FHP. Studies have suggested that vibration stimulus is capable of improving joint proprioception. Thus the aim of this study was to investigate the effects of one session of whole body vibration (WBV) training in standing position on cervical joint position sense in individuals with FHP. Thirty (12 females and 18 males) individuals with FHP were randomly assigned to a vibration (experimental) or non-vibration (control) group. The vibration group experienced 5 sets of WBV while holding the head and neck in retraction. The non-vibration group held the same position for the same time without any vibration. Four target angles were established to assess the absolute and constant joint position sense (JPS) er- rors for each individual. Results showed significant reduction $(\mathrm{P}<0 / 05)$ in JPS absolute errors in 2 target angles in the vibration group when compared to the non-vibration group. It was concluded that relative improvement in cervical JPS could be achieved when adding additional sensory input from WBV stimulus to the head and neck retraction exercise.

KEY WORDS: whole body vibration, joint position sense, forward head posture.

\section{Introduction}

Forward head posture (FHP) is considered to be one of the most common postural disorders in the spinal column ${ }^{1}$. Recently studies have reported impaired proprioception to be one of the results of prolonged $\mathrm{FHP}^{2,3}$. The cervical spine proprioceptive system is responsible for head orientation in space and in relation to the trunk and it is known for its role in maintaining head and neck stability ${ }^{4}$. Very high densities of muscle spindles have been observed in the deep layer of anterior and posterior craniocervical muscles $^{4}$. It has been suggested that muscle spindles play the most important role in providing joint position sense (JPS) information to the central nervous system (CNS). The afferent input from other mechanoreceptors in joint structures play a complementary part in providing proprioceptive information for the CNS. Clinicians have investigated the effects of different training protocols on proprioception acuity with the aim of activating proprioceptive structures in the cervical spine. Some of these rehabilitative programs have shown to improve cervical proprioception while others indicating no significant improvement ${ }^{5,6}$. Jull et al. (2007) compared the effects of two proprioception training exercises including eye-head-coordination exercise which is designed to target deep sub occipital muscles and crania-cervical flexion exercise with the aim of activating deep cervical flexors ${ }^{7}$. The results of this study indicated that both of these training methods lead to improvement of JPS but there were no significant difference in improving proprioception between the methods. Furthermore the results of this study implied that despite the training protocol, it seems that the underlying mechanism responsible for the observed effects on reposition acuity is related mostly to the improvement of cervical afferent input from the highly impacted muscle spindles in the deep 
cervical muscles. Our review of literature indicated that studies regarding proprioceptive training have been investigated mostly on patients suffering from neck pain or whiplash injury and no previous study has assessed the efficacy of a proprioception approach on individuals with forward head posture.

Whole body vibration (WBV) as an efficient neuromuscular training method for athletes and patients has become popular over the last decades. However there are some inconsistencies regarding the effects of WBV. Some studies have reported fatigue and reduced cutaneous sensations to be the possible risks of WBV ${ }^{8,9}$. The duration of the vibration stimulus seems to play an important role in the development of these complexities. On the other hand numerous studies have reported beneficial adaptations as a result of repeated or acute exposure to WBV. Increased bone density, muscle strength, power, flexibility, motor performance and balance are among the effects of WBV on different orthopedic aspects ${ }^{10-13}$. Few studies have suggested that vibration stimulus is capable of improving joint proprioception ${ }^{14-16}$. It has been hypothesized that vibration is able to stimulate the muscle spindles which results in altered motor neuron excitability and reflex activation of muscles known as tonic vibration reflex ${ }^{17}$.

To date, studies regarding the effect of WBV on proprioception have focused mostly on lower body joints. There have been only few studies investigating the effects of WBV on upper body joint proprioception, with no study exploring the cervical region. However there is only one published study which observed significant increase in neuromuscular activity levels of neck muscles during exposure to WBV ${ }^{18}$. According to the findings of this study, with specific vibration settings, there is a great chance that vibration could reach and effect the cervical region. Thus the aim of this study was to investigate the effect of one session of whole body vibration training in standing position on cervical joint position sense in individuals with FHP.

\section{Material and method}

Thirty (12 females and 18 males) individuals with FHP, between the ages of 19 and 27 participated in the study from the Rehabilitation School of Shahid
Beheshti University of Medical Science. They were randomly assigned to either a vibration or non-vibration group (Tab. I).

Inclusion criteria for selecting individuals with FHP were based on a craniovertebral angle (CVA) less than 48 degrees $^{19}$. The CVA is the angle between the horizontal line passing through $\mathrm{C} 7$ and a line extending from the tragus of the ear to $C 7^{19}$. In order to assess the head posture, subjects were asked to stand in a self-selected upright postur ${ }^{19}$. Markers were placed on the skin overlying the spinous process of the $\mathrm{C} 7$ and the tragus of the ear ${ }^{19}$. A photo was taken from the side view with the Galaxy Tab A (SM-P555) camera. The CVA was calculated using the ON 2D Measurement application on the Galaxy Tab A (SM-P555) tablet. Other eligibility criteria for selecting subjects were based on both the contradiction criteria for WBV and cervical proprioception examination, which included having no history of neck pain, fracture, neuromuscular disorder, chronic headache, whiplash injury, thoracic kyphosis and sway back, epilepsy, gallstone and kidney stone, joint implants, balance disorders, cardiovascular diseases, recent wounds and inflammatio ${ }^{9}$. Participants were instructed to avoid any caffeine, sleeping pills or any drug that stimulates the central nervous system 48 hours before the test. In order to avoid the effect of fatigue, they were reminded not to do any hard work out prior to the examination. The procedure and purpose of the study were explained to the individuals and all of them signed a written consent form prior to the test. This study was approved by Ethics Committee Board of the Faculty of Rehabilitation of Shahid Behetshi University of Medical Science.

\section{Joint position sense testing}

Cervical JPS was assessed with the cervical range of motion instrument (CROM 3 Deluxe, Performance Attainment Associates manufacture, USA) by utilizing an active reproduction of active positioning. The JPS testing protocol contained relocation of 4 target angles which were selected randomly for each subject. $50 \%$ of flexion range of motion (ROM), $50 \%$ of extension ROM, adapted head posture (AHP) and corrected head posture (CHP) were the selected target angles. The AHP was defined as the subject's habitual posture when sitting in the upright position. The CHP

Table I. Participants' demographics.

\begin{tabular}{lll}
\hline Group & Vibration & Non-vibration \\
\hline Number of participants & 15 & 15 \\
gender & 9 males 12 females & 9 males 12 females \\
Age (year) & $20.80 \pm 2.14$ & $22 \pm 2.13$ \\
Height $(\mathrm{cm})$ & $174.60 \pm 9.99$ & $170 \pm 9.30$ \\
Weight $(\mathrm{kg})$ & $71.80 \pm 17.25$ & $68.53 \pm 16.23$ \\
\hline
\end{tabular}


in this study was the ability of the subjects to reproduce the CVA of 50 degrees. To begin testing, subjects were seated with their lumbar and lower thoracic region supported by the back of the chair. In order to determine their target angle, subjects were asked to lean back comfortably, look forward and keep their head and neck in their habitual self- selected position. The examiner then recorded the angle showing on the device as their AHP. Next they were instructed to first perform a retraction of the head and neck and then bend their head and neck forward and backward as far as possible and again the examiner recorded their flexion and extension ROM. This procedure was repeated 3 times. After establishing the target angles, subjects were asked to close their eyes. For each target angle they had to slowly and actively move their head towards the target angle. With the verbal command of the therapist they noticed they had reached the target angle. Then they were asked to focus on it and memorized it for 10 seconds and with the instruction of the examiner actively relocate the target angle. In order to relocate the 50 degrees of the CVA they were instructed to do a head retraction which is the corrected head posture. In order for the participants to fully establish the posture they were asked to tuck their chin down, left up the back of the head and slide the head backward as if closing a file drawer. The aim was to enable the posterior translation of the head in the sagittal plane with zero sagittal rotation. This was the same instruction given to the participants later on while standing on the WBV platform. At the same time the participants were trying to establish the CHP the examiner monitored their CVA in real time with the use of the ON 2D Measurement application on the Galaxy tablet. When the subjects reached the 50 degrees of CVA, they were asked to hold and memorize it for $10 \mathrm{sec}-$ onds and then relocate it. The purpose of choosing to relocate the CVA of 50 degrees was that, this angle is considered to be the normal and neutral posture of the head and neck ${ }^{19}$. Each relocation trial was repeated 6 times and the mean absolute and constant JPS errors were calculated for each target angle.

\section{Whole body vibration training protocol}

After recording the baseline variables, the participants were instructed to stand on the vibration platform bare footed and distribute their weight evenly on both feet. Next they were asked to do a head and neck retraction while looking forward with their knees at 30 degrees of flexion ${ }^{20}$. In order to prevent fatigue in the subjects cervical area due to holding their head and neck in retraction, they were asked to hold this position for 15 seconds then rest for 5 seconds. Since each set lasted 60 second this allowed the subjects to do 3 retractions in each set. In total each subject performed 15 retractions during the training. The vibration group experienced vertical vibration at a frequency and amplitude of $30 \mathrm{~Hz}$ and $4 \mathrm{~mm}$ during the sets and the non-vibration group maintained their given position without vibration ${ }^{9}$. Immediately after the intervention, the same JPS testing from the baseline was performed and the difference between the mean of the variables were analyzed.

\section{Data analysis}

All statistical analysis were performed using the SPSS 18.0 software. A Kolmograph-Smirnov (K-S) test was used to determine the normality of each variable. An independent t-test was used to compare the mean of constant and absolute errors between the groups. Alpha was set at 0.05 .

\section{Result}

The entire data regarding the joint position sense testing is shown in Table II and Figures 1, 2. Although the vibration group in most angles showed better results at repositioning the target angles, the independent t-test revealed no significant difference for constant errors between the vibration and non-vibration group. However the absolute errors of repositioning the AHP ( $p=0.04)$ and the $50 \%$ of extension ROM ( $p=0.001)$ were significantly less in the vibration group compared to the non-vibration group.

Table Il. Joint position sense constant errors in the vibration and non-vibration groups (mean \pm standard deviation).

\begin{tabular}{|c|c|c|c|c|}
\hline $\begin{array}{l}\text { Target angle } \\
\text { (degree) }\end{array}$ & & $\begin{array}{l}\text { Vibration } \\
N=15\end{array}$ & $\begin{array}{l}\text { Non-vibration } \\
\mathrm{N}=15\end{array}$ & $\begin{array}{l}P \\
\text { value }\end{array}$ \\
\hline $\mathrm{AHP}$ & $\begin{array}{l}\text { Pre test } \\
\text { Post test }\end{array}$ & $\begin{array}{l}1.50 \pm 2.85 \\
1.34 \pm 1.78\end{array}$ & $\begin{array}{l}3.90 \pm 1.19 \\
-0.23 \pm 3.18\end{array}$ & 0.49 \\
\hline $\mathrm{CHP}$ & $\begin{array}{l}\text { Pre test } \\
\text { Post test }\end{array}$ & $\begin{array}{l}1.53 \pm 2.85 \\
1.45 \pm 3.89\end{array}$ & $\begin{array}{l}3.16 \pm 1.57 \\
3.20 \pm 1.36\end{array}$ & 0.09 \\
\hline $50 \%$ of flexion ROM & $\begin{array}{l}\text { Pre test } \\
\text { Post test }\end{array}$ & $\begin{array}{l}1.30 \pm 2.30 \\
2.14 \pm 2.67\end{array}$ & $\begin{array}{l}-0.41 \pm 2.95 \\
0.26 \pm 3.29\end{array}$ & 0.11 \\
\hline $50 \%$ of extension ROM & $\begin{array}{l}\text { Pre test } \\
\text { Post test }\end{array}$ & $\begin{array}{l}1.82 \pm 2.95 \\
1.57 \pm 1.14\end{array}$ & $\begin{array}{l}1.17 \pm 3.75 \\
1.03 \pm 5.01\end{array}$ & 0.98 \\
\hline
\end{tabular}




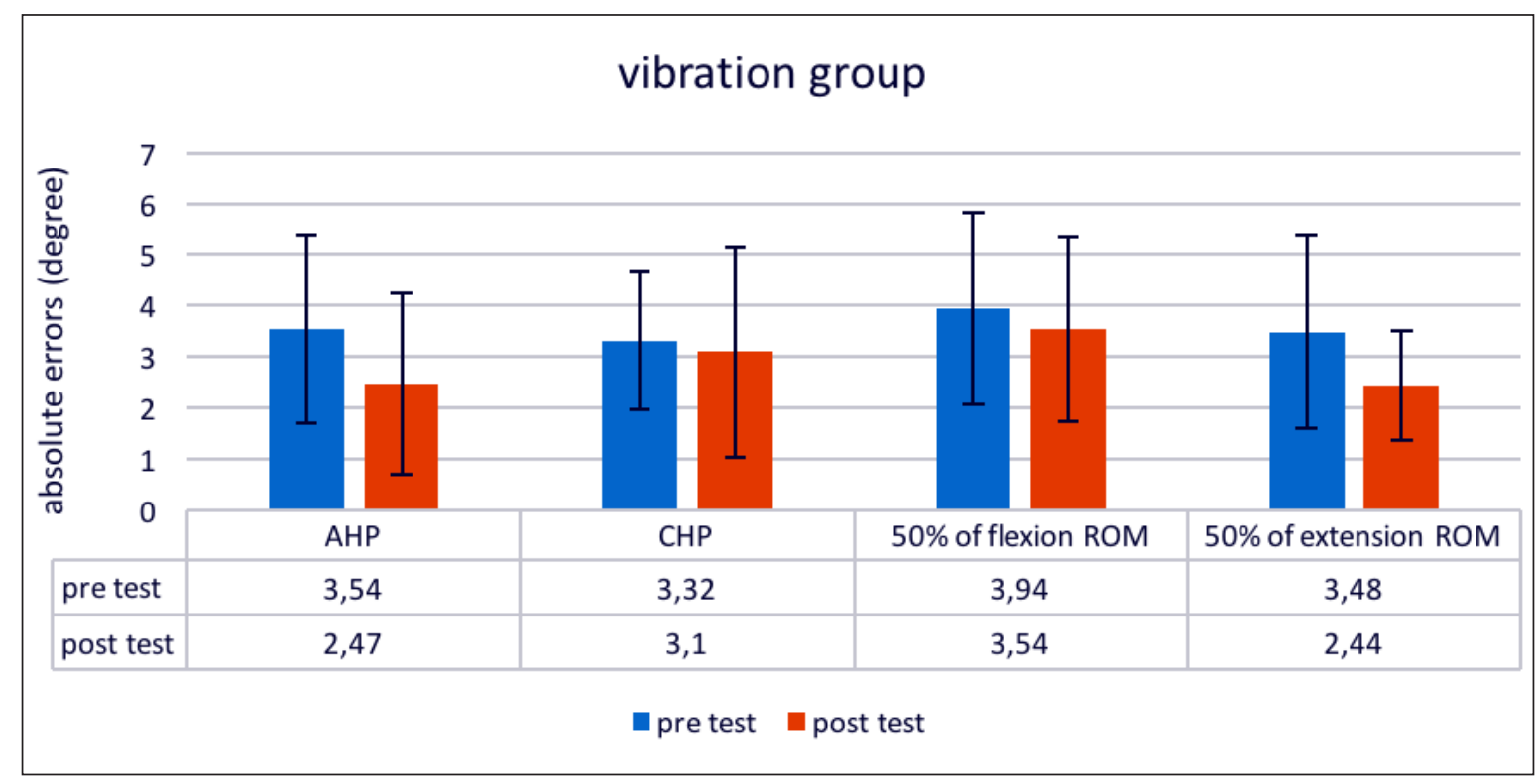

Figure 1. Mean + SD of absolute repositioning errors pre and post treatment in 4 target angles. The decrease in absolute errors in the adapted head posture (AHP) and the $50 \%$ of extension ROM target angles were associated with a significant change $(\mathrm{P}<0.05)$. CHP, Corrected Head Posture; AHP, Adapted head posture.

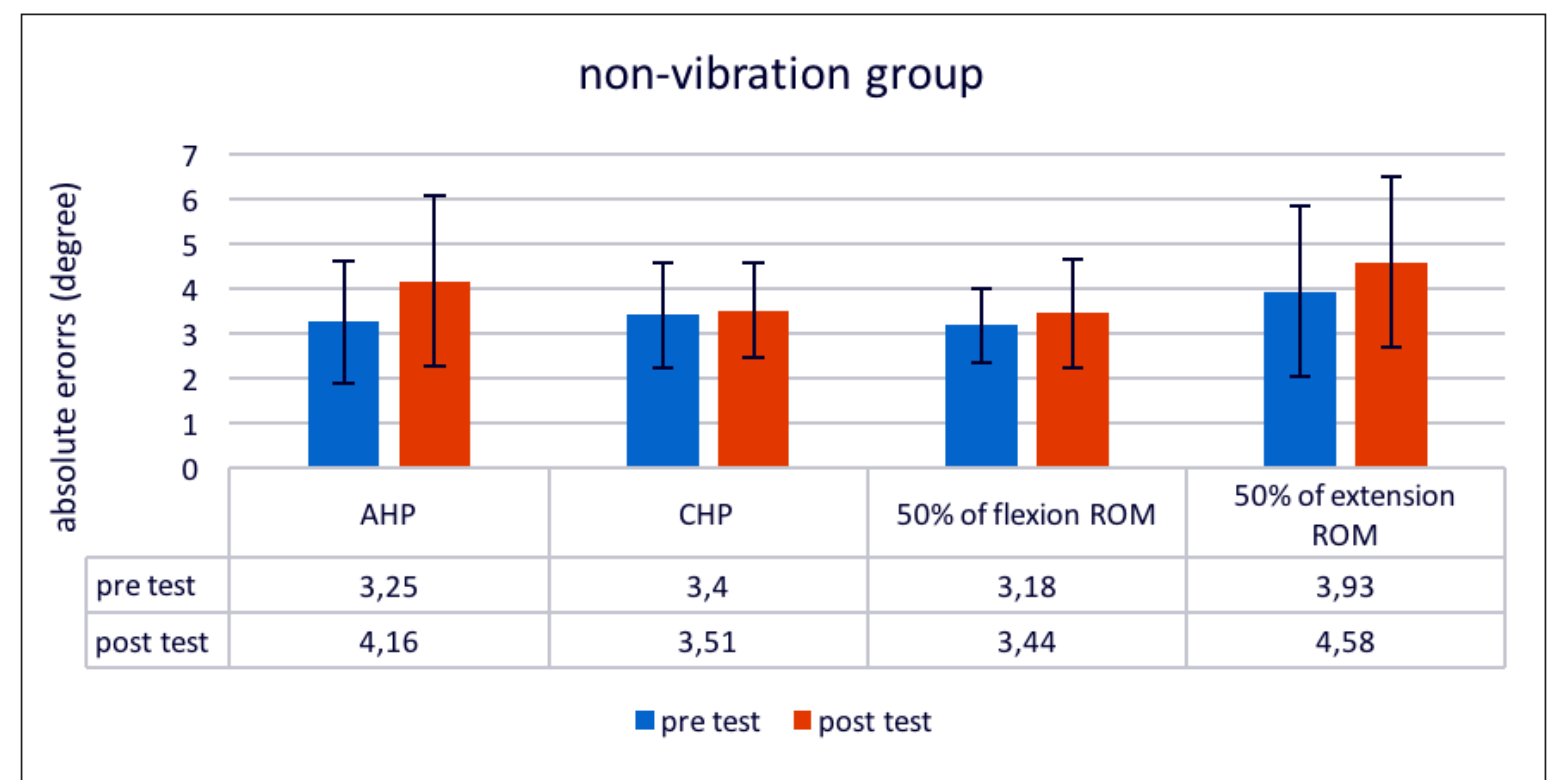

Figure 2. The mean + SD of absolute repositioning errors pre and post treatment in the non-vibration group. There was no significant change in the absolute errors of the 4 target angles in the non-vibration group ( $P>0.05)$. CHP, Corrected Head Posture; AHP, Adapted head posture.

\section{Discussion}

This is the first study to investigate the effect of WBV on head and neck joint position sense. We found that in individuals with FHP, 5 minute exposure to WBV, had significant effects on JPS accuracy in some target angles. AHP and 50\% of extension ROM target angles were the most responsive to WBV. The results observed in this study are consistent with the findings demonstrated in some previous studies. Both
Fontana et al. (2005) and Tripp et al. (2009) investigated the immediate effects of WBV on lumbosacral and elbow joint position sense and observed enhancement of JPS acuity ${ }^{14,16}$. Lee et al. (2013) measured the repositioning error of the lumbar neutral upright posture and also monitored the coordination between the pelvic and lumbar spine during a functional reached test after short exposure to $\mathrm{WBV}^{15}$. Their results indicated that WBV had some effects on repositioning acuity since the decrease observed in JPS er- 
rors was not statistically significant. However the significant improvement in lumbo-pelvic coordination during the functional reach test was an indicator of improved proprioception in dynamic motion.

There have been some explanations for the underlying mechanism by which WBV can effect neuromuscular responses. Changes induced by the vibration within the muscles alters the intrafusal fiber tension leading to la-afferent stimulation which results in reflex contraction of the muscles ${ }^{17,11}$.

Tonic vibration reflex through activation of poly synaptic pathways and muscle spindles can increase the recruitment of motor unites along with agonist and antagonist coactivation ${ }^{17,11}$. Altogether these responses could result in increased muscle stiffness and activity which are known to have a positive effect on joint proprioception.

However some previous studies found little or no effects regarding the acute effects of WBV on proprioception. Pollack et al. (2011) investigated the effect of WBV on knee and ankle joint position sense on healthy individuals and there results showed no meaningful changes afterwards ${ }^{9}$. The results of Hong et al. (2010) also revealed that acute bouts of shoulder WBV in a push up position had no significant effects on JPS acuity in healthy individuals ${ }^{21}$.

Studies mentioned having contrasting results with the present study have involved healthy people without any known pathologies or postural impairments. Whereas in the present study we included individuals with postural defect and associated proprioceptive impairment which has been mentioned by other studies $^{2,3}$. Improvements in knee joint position sense in people with ligament injuries and enhancement in movement detection threshold in those with knee arthritis have been reported by other studies after repeated exposure to WBV ${ }^{12,13}$. This supports the possibility of greater effects of WBV on proprioception in individuals with known proprioception impairments.

Also the contrasting findings of these studies may be explained by the use of different vibration settings (amplitude and frequency) and holding a relaxed or static posture or performing dynamic exercises while experiencing WBV20.

Regarding the effects of different types of contraction on neuromuscular activation during WBV, Abercromby et al. (2007) observed the greatest neuromuscular response during isometric and concentric contractions $^{20}$. Any change in the muscle's length during voluntary muscle contraction increases the la afferent sensitivity by altering the intrafusal fiber tensions. Other studies have also mentioned that increased muscle pre-activation may lead to greater responses to WBV22. In the study done by Pollack et al. (2011) on knee and ankle JPS the subjects were asked to stand on the vibration platform with their knees being straight without any degrees of knee flexion ${ }^{9}$. The subjects in the study of Lee et al. (2013) were also instructed to stand in there relaxed upright position on the WBV platform without performing any kind of lumbar activity during vibration ${ }^{15}$. Whereas in the present study we added neck retraction during WBV, which is the flexion of the upper cervical and extension of the lower cervical segments ${ }^{23}$. This exercise is known to strengthen deep neck flexors and stretch the upper cervical extensors. Both of these groups of muscles are highly impacted by muscle spindles and their weakness and length imbalance in individuals with forward head posture has been reported by other studies $^{24}$. These muscles are also responsible for the head and cervical stability. In the present study the subject's ability to identify the $50 \%$ of extension and flexion ROM target angles improved after WBV but was only significant in the extension target angle. The suboccipital, splenius and semi splenius muscles are mostly responsible for carnio-cervical extension 25 . In the study done by Perchthaler et al. (2015) significant increase in the EMG activity of upper erector spine muscles and splenius muscle during WBV were indicated $^{18}$. The overall JPS error decreased in the CHP and AHP target angles following WBV, but was only significant in the AHP target angle. This finding implies that vibration together with the use of head and neck retraction may be beneficial for the better identification of the CNS of the cervical joint positions. However since the CHP seems to be unfamiliar for the CNS and since forward head posture has been present for a long time, WBV had the most effect on the AHP target angle. Further sessions of training may be needed to give a clearer picture of the CHP to the CNS.

As in all studies, the present study had a few limitations and recommends new areas for future investigations. In the present study we examined the immediate effects of WBV on cervical JPS. However the effects of more training sessions should be taken in to consideration as well. Additionally, the individuals only experienced WBV in the standing position. Therefore the effects of different body positions should be investigated as different results may be obtained regarding cervical proprioception.

\section{Conclusion}

The findings of this study suggest that relative improvement in cervical JPS can be achieved when adding additional sensory input from WBV stimulus to the head and neck retraction exercise.

\section{Acknowledgement}

The Authors would like to thank all the participants for their great cooperation.

\section{Ethics}

The Authors declare that this research was conducted following basic ethical aspects and international standards as required by the journal and recently update in ${ }^{26}$. 


\section{References}

1. Griegel-Morris $\mathrm{P}$, Larson $\mathrm{K}$, Mueller-Klaus $\mathrm{K}$, Oatis CA. Incidence of common postural abnormalities in the cervical, shoulder, and thoracic regions and their association with pain in two age groups of healthy subjects. Physical therapy. 1992;72 (6):425-431.

2. Lee M-Y, Lee H-Y, Yong M-S. Characteristics of cervical position sense in subjects with forward head posture. Journal of physical therapy science. 2014;26(11):1741-1743.

3. Shaghayegh-Fard B, Ahmadi A, Ma'roufi N, Sarraf-Zadeh J. The evaluation of cervical position sense in forward head posture subjects and compares it with normal subjects. Archives of Rehabilitation. 2015;16(1):48-57.

4. Kulkarni V, Chandy M, Babu K. Quantitative study of muscle spindles in suboccipital muscles of human foetuses. Neurology India. 2001;49(4):355.

5. Armstrong BS, McNair PJ, Williams M. Head and neck position sense in whiplash patients and healthy individuals and the effect of the cranio-cervical flexion action. Clinical Biomechanics. 2005;20(7):675-684.

6. Revel M, Minguet M, Gergoy P, Vaillant J, Manuel JL. Changes in cervicocephalic kinesthesia after a proprioceptive rehabilitation program in patients with neck pain: a randomized controlled study. Archives of physical medicine and rehabilitation. 1994;75(8):895-899.

7. Jull G, Falla D, Treleaven J, Hodges P, Vicenzino B. Retraining cervical joint position sense: the effect of two exercise regimes. Journal of Orthopaedic Research. 2007;25(3):404412.

8. Bongiovanni LG, Hagbarth KE, Stjernberg L. Prolonged muscle vibration reducing motor output in maximal voluntary contractions in man. The Journal of physiology. 1990 Apr 1;423 (1):15-26.

9. Pollock RD, Provan S, Martin FC, Newham DJ. The effects of whole body vibration on balance, joint position sense and cutaneous sensation. European journal of applied physiology. 2011;111(12):3069-3077.

10. Cerciello S, Rossi S, Visonà E, Corona K, Oliva F. Clinical applications of vibration therapy in orthopaedic practice. Muscles Ligaments Tendons Journal. CIC Edizioni Internazionali; 2016 May 19;6(1):147-156.

11. Cardinale $M, B o s c o ~ C$. The use of vibration as an exercise intervention. Exercise and sport sciences reviews. 2003;31(1): 3-7.

12. Moezy A, Olyaei G, Hadian M, Razi M, Faghihzadeh S. A comparative study of whole body vibration training and conventional training on knee proprioception and postural stability after anterior cruciate ligament reconstruction. British journal of sports Medicine. 2008;42(5):373-385.

13. Aaboe J, Henriksen M, Christensen R, Bliddal H, Lund H. Effect of whole body vibration exercise on muscle strength and proprioception in females with knee osteoarthritis. The Knee.
2009;16(4):256-261.

14. Tripp BL, Faust D, Jacobs P. Elbow joint position sense after neuromuscular training with handheld vibration. Journal of athletic training. 2009;44(6):617-623.

15. Lee $T$, Chow $D$, editors. Effects of whole body vibration on spinal proprioception in normal individuals. Engineering in Medicine and Biology Society (EMBC), 2013 35th Annual International Conference of the IEEE; 2013: IEEE.

16. Fontana TL, Richardson CA, Stanton WR. The effect of weightbearing exercise with low frequency, whole body vibration on lumbosacral proprioception: A pilot study on normal subjects. Australian Journal of Physiotherapy. 2005;51(4): 259-263.

17. De Gail P, Lance J, Neilson P. Differential effects on tonic and phasic reflex mechanisms produced by vibration of muscles in man. Journal of neurology, neurosurgery, and psychiatry. 1966;29(1):1.

18. Perchthaler D, Hauser S, Heitkamp H-C, Hein T, Grau S Acute effects of whole-body vibration on trunk and neck muscle activity in consideration of different vibration loads. Journal of sports science \& medicine. 2015;14(1):155.

19. Nemmers TM, Miller JW, Hartman MD. Variability of the Forward Head Posture in Healthy Community-dwelling Older Women. Journal of geriatric physical therapy. 2009;32(1):1014.

20. Abercromby AF, Amonette WE, Layne CS, Mcfarlin BK, Hinman MR, Paloski WH. Variation in neuromuscular responses during acute whole-body vibration exercise. Medicine \& Science in Sports \& Exercise. 2007;39(9):1642-1650.

21. Hong J, Velez M, Moland A, Sullivan J. Acute effects of whole body vibration on shoulder muscular strength and joint position sense. Journal of human kinetics. 2010;25:17-25.

22. Roelants M, Verschueren SM, Delecluse C, Levin O, Stijnen V. Whole-body-vibration-induced increase in leg muscle activity during different squat exercises. Journal of Strength and Conditioning Research. 2006;20(1):124.

23. Ordway NR, Seymour RJ, Donelson RG, Hojnowski LS, Edwards WT. Cervical flexion, extension, protrusion, and retraction: a radiographic segmental analysis. Spine. 1999;24(3): 240-247.

24. Gong W, Kim C, Lee Y. Correlations between cervical lordosis, forward head posture, cervical ROM and the strength and endurance of the deep neck flexor muscles in college students. Journal of Physical Therapy Science. 2012;24(3):275-277.

25. Rezasoltani A, Nasiri R, Faizei A, Zaafari G, Mirshahvelayati A, Bakhshidarabad $\mathrm{L}$. The variation of the strength of neck extensor muscles and semispinalis capitis muscle size with head and neck position. Journal of bodywork and movement therapies. 2013;17(2):200-203.

26. Padulo J, Oliva F, Frizziero A, Maffulli N. Muscles, Ligaments and Tendons Journal - Basic principles and recommendations in clinical and field science research: 2016 update. MLTJ. 2016;6(1):1-5. 